\title{
Preamplified photodetectors for high-fidelity postselecting optical devices
}

\author{
John Jeffers \\ SUPA, Department of Physics, University of Strathclyde, \\ John Anderson Building, 107 Rottenrow, Glasgow G4 ONG, U.K.
}

\begin{abstract}
The fidelity of postselecting devices based on direct photon number detection can be significantly improved by insertion of a phase-insensitive optical amplifier in front of the detector. The scheme is simple, and the cost to the probability of obtaining the appropriate detector outcome is low.

PACS numbers: 03.67.-a, 42.50.Dv, 42.50.-p, 42.79.-e
\end{abstract}

\section{INTRODUCTION}

The reliable production of tailored quantum states is one of the main challenges in quantum information. Experiments in optics rely on postselection to do this $[1,[2,3]$. A general perfect postselecting device is shown in Fig. 1] A multi-component quantum state is fed into a device which transforms the input state. One of the outputs (arm 2) is measured, and when the measurement gives a particular result (represented mathematically by a probability operator measure element $\hat{\pi}_{\mathrm{c}}$ ) the device produces the correct useful state $\hat{\rho}_{\mathrm{C}}$ in arm 1. If one of a set of incorrect measurement results $\left(\hat{\pi}_{\mathrm{i} j}\right)$ is found the device produces an incorrect state $\hat{\rho}_{\mathrm{i} j}$.

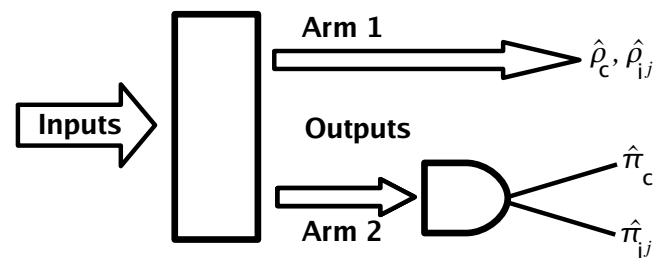

FIG. 1: A general postselecting device.

Often in optics the unselected state is mixed, so postselection is essential. Simple examples include the generation of heralded single photon states from twin-beam parametric downconversion 2], or the quantum scissors device [3], which produces a superposition of zero and one photon. Interest in the topic received a further boost with the realisation that it might be possible to perform scalable linear optical quantum computing, with both state production and logic gate operation relying on postselection [4, 5, 6]. Variations on this include the one-way quantum computer, which produces states from measurements performed on cluster states 7].

Imperfections, either in internal device components or in detection, are a serious problem for real postselectors. As a result they do not make the state that they would have made if they had been functioning perfectly. Photodetectors are poor at present, suffering from low quantum efficiency, from dark counts and from an inability to distinguish between higher photon numbers. This means that the single photon number readout from a detector, which ought to correspond to a pure number-state measurement, in fact corresponds to a mixed measured state
8]. The effect of this on the postselector output state is to mix $\hat{\rho}_{\mathrm{C}}$ with the set of incorrect states $\hat{\rho}_{\mathrm{i} j}$.

The quality of device output can be quantified by the fidelity, which is a measure of the closeness of the imperfect output state produced, $\hat{\rho}_{\mathrm{c}}^{\prime}$, to the required output state, $\hat{\rho}_{\mathrm{C}}$. The standard definition, if $\hat{\rho}_{\mathrm{C}}$ is pure, is $F=\operatorname{Tr}\left(\hat{\rho}_{\mathrm{c}} \hat{\rho}_{\mathrm{c}}^{\prime}\right)$, which is unity for a perfect device 9$]$.

Optical quantum information processors, which might be composed of thousands of postselectors, require extremely high fidelities. The improvement of photodetectors to such levels is unrealistic, but there have been proposals for novel detection schemes to improve fidelity. For example, photon added homodyne detection [10] does this, but with a high cost to the probability of detecting the required state (reduced by more than 100-fold if the fidelity is to be greater than 0.99). Another proposed approach is to use a nonlinear optical material formed by atoms in a dielectric waveguide to perform quantum nondemolition detection of photon number 11]. This scheme has the appealing feature that the detected photons can be re-used. Despite this, both schemes are much more complicated than direct detection, and the former requires photon number states as a resource.

Here a scheme is proposed which uses inefficient direct photodetection preceded by a phase-insensitive optical amplifier. Amplifiers are not typically used in quantum optical experiments as they add noise photons [12], which swamp any quantum characteristics of the amplified state 13]. They have been used to offset detector inefficiency, improving the signal to noise ratio for direct detection autocorrelation measurements of laser light [14], but never for quantum states. However, there have been recent improvements to amplifiers for quantum systems [15]. Also, it has been shown, using retrodictive quantum theory [16], that the state transformation made by an amplifier of gain $G$ forwards in time is the same as that made by an attenuator with transmission $1 / G$ backwards in time and vice versa 17], which leads to seemingly strange input photon number expectation values 18].

In the next section the retrodictive conditional probability is introduced as a measure of fidelity appropriate for optical postselecting devices. Then results are provided for postselection based on recording zero or one photocount, followed by a simple explanantion. An analysis of the cost of amplification follows, in terms of a reduction in the photocount probability. In the final sec- 
tion the results are discussed and conclusions presented.

\section{DEVICE FIDELITY BASED ON PREAMPLIFIED DETECTION}

\section{A. Conditional probability as a fidelity measure}

For a pure state postselector with perfect internal components, based on detecting photon number states, substitution of $\hat{\rho}_{\mathrm{c}}$ and $\hat{\rho}_{c}^{\prime}$ allows the fidelity to be expressed as a sum of terms [19]

$$
F=P^{\mathrm{r}}(\mathrm{c} \mid \mathrm{c})+\sum_{j} P^{\mathrm{r}}(\mathrm{i} j \mid \mathrm{c}) \operatorname{Tr}_{1}\left[\hat{\rho}_{\mathrm{c}} \hat{\rho}_{\mathrm{i} j}\right]
$$

Here $P^{r}(\mathrm{c} \mid \mathrm{c})$ is the retrodictive conditional probability that the number of photons in the measurement arm (2) is the same as that indicated by the detector, and $P^{\mathrm{r}}(\mathrm{i} j \mid \mathrm{c})$ is one of the set of probabilities that the number of photons is different from that indicated by the detector. The first term, which we denote $F_{\mathrm{r}}$, has been proposed as a simple measure of fidelity [19]. It has advantages over $F$. Firstly it depends only on measurement arm properties: the 'prior' probabilities [20] of detectable states in the measurement arm and the properties of the mixing performed there. Secondly, it is the natural quantity to maximise in order to enhance fidelity (if $F_{\mathrm{r}}$ is unity the confidence in the detector result is perfect, as is $F$ ) [21]. Often the overlaps between the correct output state and the incorrect ones will be small. Furthermore, as the detection scheme is improved, the probabilities that the measured state is not that indicated by the detector diminish. Thus $F_{\mathrm{r}}$ forms a close lower bound on $F$.

The detector is characterised by an efficiency $\eta$, and discriminates between different photon numbers. This is not typical, but detectors which discriminate between zero, 1 and more than 1 photon exist [22], and here postselection based solely on recording zero or 1 count is considered, as this is the most prevalent. For practical purposes then the detector is equivalent to a perfect discriminating device preceded by an attenuator of transmission $\eta$ [23]. The detection system is completed by an ideal amplifier of gain $G$ (Fig. 2) which adds the minimum amount of noise. Discussion of both extra amplifier noise and dark counts is postponed until later. The fictitious attenuator and real amplifier jointly form a compound mixing device preceding the perfect detector [19].

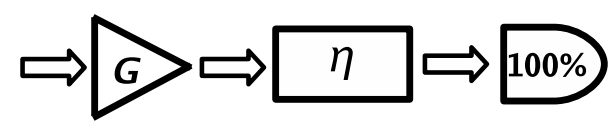

FIG. 2: An amplifier precedes the imperfect detector, modelled by an attenuator in front of a perfect detector.

Retrodictive conditional probabilities can be found from Bayes' Theorem. If the postselecting device is supposed to produce the correct state when $n$ photons are detected then $F_{\mathrm{r}}$ is

$$
F_{\mathrm{r}}(n)=\frac{p_{n} P^{\mathrm{p}}(n \mid n)}{\sum_{m} p_{m} P^{\mathrm{p}}(n \mid m)}
$$

where $p_{m}$ is the prior probability of $m$ photons in the measurement arm, and $P^{\mathrm{p}}(n \mid m)$ is the predictive conditional probability that $n$ photons exit the compound mixing device and are recorded as counts at the perfect detector, given that $m$ enter it. The required probabilities can be straightforwardly calculated. The prior probabilities are the diagonal elements, in the photon number basis, of the arm 2 state formed by tracing the joint output state of the two arms over arm 1. The conditional probabilities are well-known from the quantum theories of the amplifier and attenuator [18, 24],

$$
P^{\mathrm{p}}(n \mid m)=\sum_{q=n}^{\infty}\left(\begin{array}{c}
q \\
n
\end{array}\right) \eta^{n}(1-\eta)^{q-n}\left(\begin{array}{c}
q \\
m
\end{array}\right) \frac{(G-1)^{q-m}}{G^{q+1}}
$$

The denominator in $F_{\mathrm{r}}(n)$ is the probability that $n$ counts are recorded, which is also altered by the amplifier.

\section{B. Postselection based on zero or one photocount}

First postselection based on recording 0 photocounts is examined. The prior photon number probability distribution will be fixed by the particular device under consideration, but a distribution must be chosen for calculation purposes. If all of the prior photon number probabilities

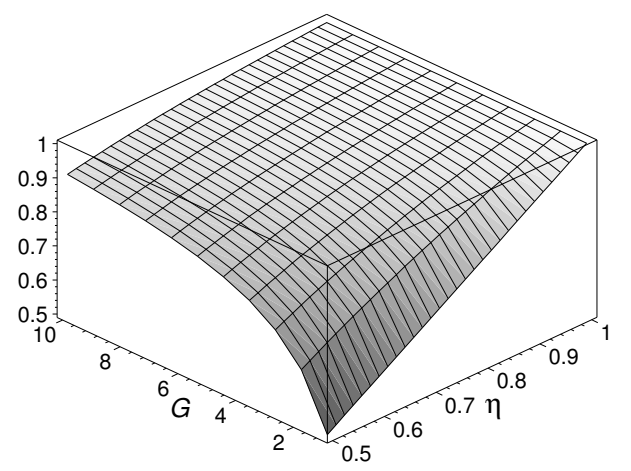

FIG. 3: Fidelity against $G$ and $\eta$ for postselection based on 0 counts, for each photon number in the measurement arm having equal prior probability.

are equal Fig. 3 is obtained, which shows $F_{\mathrm{r}}(0)$ as a function of $\eta$ and $G$. The greater the gain, the greater is the improvement over the no-amplifier, $G=1$ limit [25]. The device will show increase in fidelity as the gain increases for any $\left(p_{0} \neq 0\right)$ prior probability distribution. A simple example might be a two-photon state generator formed by a single photon input into each input arm of a 50/50 beam splitter. If no photons are detected in one output arm then two are produced in the other. For this device all of the prior probabilities vanish except $p_{0}=p_{2}=1 / 2$. 


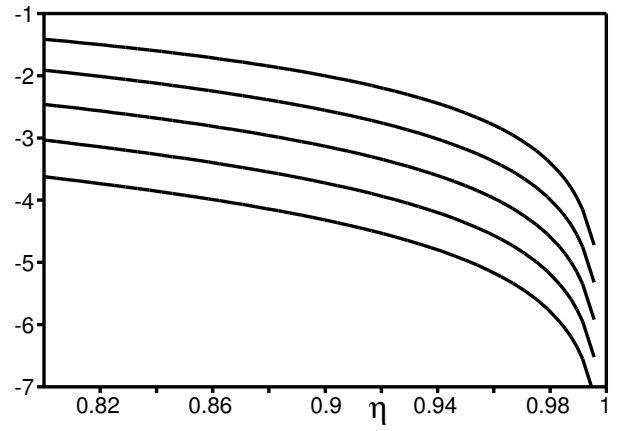

FIG. 4: $\log _{10}\left(1-F_{\mathrm{r}}(0)\right)$ against $\eta$ for the two-photon state generator. The lines are for (from top to bottom) $G=1,2,4$, 8 and 16.

A plot of the log of the difference between the fidelity and 1 against $\eta$ is shown in Fig. 4 for various amplifier gains. Here as $G$ is increased the fidelity tends to 1 even more rapidly, a function of the particular prior probability distribution. It is possible to reach extremely high fidelities even for relatively modest gain.

For postselection based on one count, with equal prior photon number probabilities fidelity can be increased for low $G$ only if $\eta$ is below about 0.7 . The situation is different, however, if the measurement arm contains at least one photon, which is illustrated by Fig. 5] Increasing

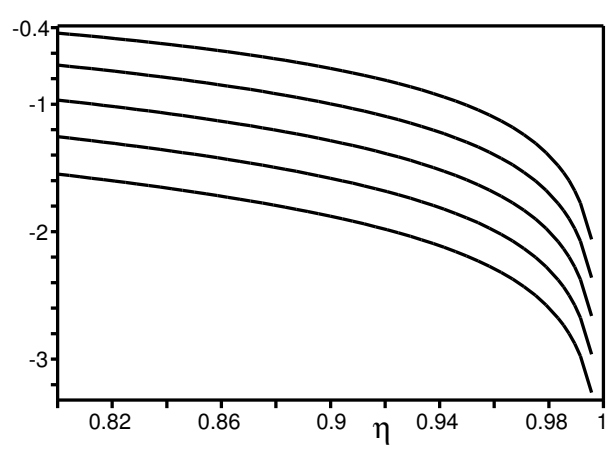

FIG. 5: $\log _{10}\left(1-F_{\mathrm{r}}(1)\right)$ against $\eta$ for equal prior probability of each nonzero photon number in the measurement arm. The lines are for (from top to bottom) $G=1,2,4,8$ and 16 .

the gain increases the fidelity to arbitrarily close to unity. Any prior distribution for which $p_{0}$ vanishes will show improvement in fidelity as the gain increases for all values of $\eta$.

\section{Simple explanantions for fidelity increase}

A clarification of the physics behind the effect is found by considering the measurement arm states corresponding to the measurment results. For a perfect detector these are the pure states indicated by the detector, but for an imperfect detector they are mixed [8]. Fig. [6]shows histograms of the photon number probability distribution
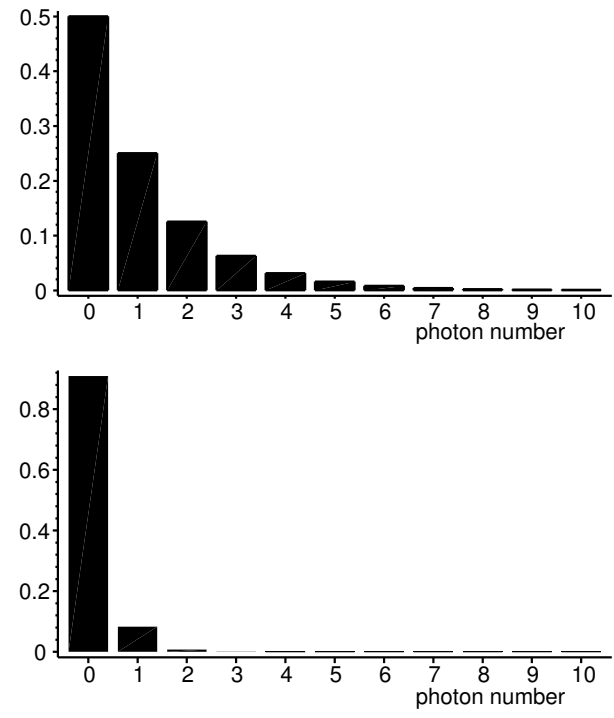

FIG. 6: Photon number probability histograms for detection of 0 photons by a detector with $\eta=0.5$. The upper histogram is the measured state if there is no amplifier present, and the lower one is for $G=10$.

of the mixed state corresponding to detection of zero photons by an imperfect detector. The effect of the amplifier (lower histogram) is to 'attenuate' the state such that the mean photon number in the projected state is $1 / G$ times the mean photon number in the projected state with no amplifier present (upper histogram) 17, 18]. This causes probability to 'pile up' at lower photon numbers, and in particular on zero.

The same effect is responsible for fidelity increase based on 1 count. For high enough gain the most likely photon number, if 1 is counted, is zero, then 1 , then 2 etc. The prior distribution then becomes important. Excluding the possibility of zero photons entering the detector amounts to omitting the zero photon component of the projected state and then renormalizing, so that 1 photon is the lowest possible photon number in the distribution. Otherwise the fidelity decreases with $G$ for high $\eta$.

An alternative view is that the amplifier shifts the photon number of the $n$-photon component of its input (the state represented by the prior distribution) from exactly $n$ to a mean of $(n+1) G-1$. In other words it separates photon numbers by a factor $G$ and adds $G-1$ photons (although the random nature of the process means that there is some overlap between the shifted distributions for different initial photon numbers). For reasonable values of $\eta$ it is very unlikely that zero counts will be obtained from a shifted 1 or 2 photon state, as it is very unlikely that so many photons will be lost at the attenuator. If zero counts are obtained, it is therefore more likely that no photon was present. 


\section{Photocount probability cost}

There is a cost associated with the large fidelity increases which are possible using preamplification, and this is seen in the photocount probabilities. The ratio of the probability that the detector records zero counts to this same probability for perfect detection quantifies this cost, and is shown in Fig. 7 (for equal prior probabilities). The curve is insensitive to $\eta$. The relative probability is

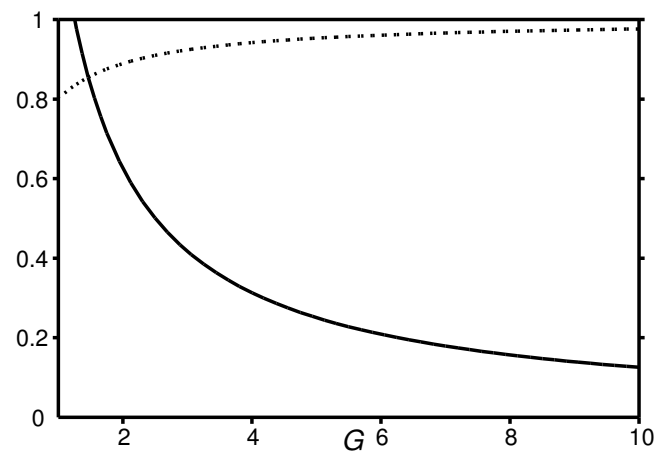

FIG. 7: Relative probability $P(0) / P(0, \eta=G=1)$ of no counts being recorded at the detector as a function of $G$ for $\eta=0.8$. Also plotted (dashed) is the fidelity.

reduced, but not excessively so. Even for $G=10$ the probability is only reduced to $1 / 8$ th of its value for perfect detection. The fidelity obtained for $\eta=0.8, G=10$ is 0.975 , but this is for a flat prior photon number distrubution (the corresponding figure for $\eta=0.9$ is close to 0.99). For states with distributions with small or vanishing probabilities of higher photon numbers the improvement can be vast, as Fig. 4 shows.

\section{CONCLUSIONS}

In this paper it has been shown that an optical preamplifier can significantly improve the fidelity of postselectors based solely on imperfect direct photodetection. A further advantage is that the amplifier does not drastically decrease the probability of device operation. Even this modest decrease in probability could be offset by including other detection results as a signature of the required number of photons in the measurement arm (e.g. if $G \eta \gg 1$ and one count is obtained the most likely photon number in the measurement arm is zero).

The scheme works best if the detected photon number is the minimum number possible in the measurement arm. Thus it is especially useful for improving the fi- delity based on detecting zero photons. For detecting single photons the improvement is almost as great, but the benefit of the method rests on the ability to produce measurement arm states which do not contain a significant vacuum component. This is a matter of postselector design and photon production technique, which is rapidly improving under the impetus of the quantum information challenge [26, 27]. For postselectors with detections in more than one output arm, such as the quantum scissors 3] amplifiers can be placed in front of each detector, and similar improvements in fidelity can be found.

Up to this point the amplifier has been assumed to add the minimum number of noise photons. The main effect of extra noise photons is to decrease the fidelity obtained for a particular gain. As was stated earlier the amplifier shifts and separates different input photon numbers, and spreads the output distributions. Extra noise spreads the distributions more, so that different photon number components of the input are not so distinguishable. This decrease in fidelity can sometimes be partially offset by increasing $G$, or the effect itself may be small because of the particular prior photon number distribution in the measurement arm. One might think that detector dark counts would cause a similar decrease in fidelity but this is not the case. Fidelity based on zero counts is unaffected by dark counts (no counts obtained $\Longrightarrow$ no dark counts obtained). Fidelity based on 1 count can be improved slightly by a nonzero dark count rate. These effects will be explored more fully in later work.

Optical amplifiers are overlooked in quantum information experiments, largely because of the necessary added noise photons, but it should be noted that these 'noise' photons are indistinguishable from amplified signal photons. Provided that the noise photons added into non signal modes can be excluded from the detection process, the photons added in the signal mode can play a useful role, and can sometimes be regarded as an additive component to the multiplicative gain $G$. For direct detection in postselectors both the quantum nature and the phase of the detected signal state are unimportant, but the result of the detection process and the confidence in that result are paramount. Amplifiers will not help the first two quantities, but can significantly improve the last.

\section{Acknowledgments}

The author thanks the Engineering and Physical Sciences Research Council for financial support and Steve Barnett and Rodney Loudon for useful discussions.
[1] H.-A. Bachor and T.C. Ralph, A Guide to Experiments in Quantum Optics, 2nd Edition, (Wiley, New York, 2004).

[2] C.K. Hong and L. Mandel, Phys. Rev. Lett. 56, 58
(1986).

[3] D.T. Pegg et al., Phys. Rev. Lett. 81, 1604 (1998); S.A. Babichev et al., Europhys. Lett. 64, 1 (2003). 
[4] E. Knill et al., Nature (London) 409, 46 (2001).

[5] T.C. Ralph et al., Phys. Rev. A 65, 012314 (2001).

[6] For a recent review article with extensive references, see P. Kok et al. quant-ph/0512071

[7] M.A Nielsen, Phys. Rev. Lett. 93, 040503 (2004); D.E Browne and T. Rudolph, Phys Rev. Lett. 95, 010501 (2005).

[8] S.M. Barnett et al., Opt. Commun. 158, 45 (1998).

[9] R. Jozsa, J. Mod. Opt. 41, 2315 (1994).

[10] A.M. Brańczyk et al., Phys. Rev. A 68, 043821 (2003) (see figs. 5 and 6 for probability cost).

[11] W.J. Munro et al., Phys. Rev. A 71, 033819 (2005).

[12] C.M. Caves, Phys. Rev. D 26, 1817 (1982).

[13] See, for example, Optical Coherence and Quantum optics, L. Mandel and E. Wolf, (Cambridge University Press, Cambridge, 1995).

[14] M. Harris et al., J. Mod. Opt. 38, 613 (1991).

[15] V. Josse et al., Phys. Rev. Lett. 96, 163602 (2006).

[16] S.M. Barnett et al., J. Mod. Opt. 471779 (2000); D.T. Pegg et al., Phys. Rev. A 66, 022106 (2002).

[17] S.M. Barnett et al., Phys. Rev. A 62, 022313 (2000).

[18] O. Jedrkiewicz et al., Phys. Rev. A 70, 033805 (2004).
[19] J. Jeffers, New J. Phys. 8, 268 (2006).

[20] D.T. Pegg and J. Jeffers, J. Mod. Opt. 52, 1835 (2005).

[21] Recently a type of state discrimination has been proposed, dubbed maximum confidence measurement, which is based on maximising this probability. See S. Croke et al., Phys. Rev. Lett. 96, 070401 (2006); P.J. Mosley et al., Phys. Rev. Lett. 97, 193601 (2006).

[22] See for example, P.P. Rohide and T.C. Ralph, J. Mod. Opt. 53, 1589 (2003).

[23] H.P. Yuen and J.H. Shapiro, IEEE Trans. Inf. Theor. 26, 78 (1980); R. Loudon, The Quantum Theory of Light, 3rd Edition (Oxford, Oxford University Press, 2000) p271ff.

[24] R. Loudon and T.J. Shepherd, Opt. Acta 31, 1234 (1984).

[25] J. Jeffers et al., in Quantum Communication, Measurement and Computing, edited by S.M. Barnett et al. (American Institute of Physics, New York, 2004).

[26] D.N. Matsukevich et al., Phys. Rev. Lett. 97, 013601 (2006).

[27] J.M. Geremia, Phys. Rev. Lett. 97, 073601 (2006). 